\title{
A morphometric study of the popliteus myotendinous complex with its clinical aspects
}

\author{
P. C. Vani, V. Raveendranath
}

Department of Anatomy, Jawaharlal Institute of Postgraduate Medical Education and Research [JIPMER], Dhanvantri Nagar, Pondicherry, India.

\section{CORRESPONDING AUTHOR:}

Raveendranath Veeramani

Department of Anatomy,

Jawaharlal Institute of Postgraduate Medical

Education and Research [JIPMER],

Dhanvantri Nagar, Pondicherry 605006,

India.

Tel: +09043997352

E-mail: dr_raveendra@rediffmail.com

DOI:

10.32098/mltj.01.2019.13

\begin{abstract}
SUMMARY
Background. The aim of the present study is to study the morphometry of the muscle-tendon complex of popliteus, and to define a relatively safe zone in posterior approach to knee or popliteal region with respect to the bony landmarks for tibial nerve and nerve to popliteus. Method. 20 cadaveric legs were used for dissection. The attachments of popliteus, length and width of tendon as well as muscle belly, were defined and noted. An imaginary line extending from the medial to lateral epicondyles of femur was taken as the intercondylar line. The distance of tibial nerve and nerve to popliteus at lower border of popliteus to both the medial and lateral epicondyle of femur was measured. Result. The attachments of popliteus to femur, capsule of knee joint, tibia and fascia over popliteus were present in all $20(100 \%)$ specimens. A distance of $65 \mathrm{~mm}$ and $40 \mathrm{~mm}$ from the medial and lateral epicondyle can be considered as safe zone for the tibial nerve and nerve to popliteus at lower border of popliteus in posterior approach to knee joint. Conclusion. Knowledge about morphometry of the popliteus and safe zone can help the orthopaedicians in reconstructive surgeries and rehabilitation procedures involving popliteal region.
\end{abstract}

\section{KEY WORDS}

popliteus muscle; nerve to popliteus; safe zone; tibial nerve

\section{INTRODUCTION}

The popliteus muscle is relatively a small and unique muscle of posterolateral corner of knee joint. The popliteal tendon, being intra-capsular, arises from anterior end of groove for popliteus, on the lateral surface of lateral femoral condyle. This tendon is joined by fibers arising from arcuate popliteal ligament, fibrous capsule, lateral meniscus, and popliteofibular ligament. As the tendon descends medially, fleshy fibers extend from its myotendinous junction and are inserted into posterior surface of tibia above the soleal line, as well as the fascia over popliteus forming floor of popliteal fossa. Popliteus activation primarily rotates the knee internally, and its tendinous bands retract the posterior arch of the lateral meniscus (1). Popliteus also unlocks the knee joint at beginning of flexion (4).

Popliteus myotendinous complex (PMTC) forms the posterolateral structures of knee along the fibular collateral ligament, arcuate ligament and posterolateral capsule. Thus, it contributes to both static and dynamic posterolateral knee joint stabilization. It also provides a greater part in preventing tibial external rotation and posterior translation during initial $30^{\circ}$ of knee flexion. Understanding the function of PMTC can also help in formulation of rehabilitation procedures for patients with posterolateral knee joint instability. The muscle is innervated by nerve to popliteus (NP), a branch of the tibial nerve $(\mathrm{TN})$, which winds around its distal border and enters the anterior surface of the muscle. The localization of nerve point and motor points of NP is important for nerve block to treat spasticity of the leg. The $\mathrm{TN}$ and NP being located posterior to knee joint are at risk of injury during surgeries of this region performed using a posterior approach.

Therefore, the present study is designed to study the morphometry of the muscle-tendon complex of popliteus and to define 
a relatively safe zone in posterior approach to knee or popliteal region with respect to the bony landmarks for TN and NP.

\section{MATERIALS AND METHODS}

The present study included 20 adult lower limbs of unknown gender obtained from the Department of Anatomy at JIPMER, Puducherry, India. The limbs with deformities and damage involving the posterior compartment of the leg were excluded from the present study. The dissection of specimens was done following the steps of Cunningham's manual of practical anatomy (8). Dissections were performed with knee in extended position. The gastrocnemius along with soleus were divided from origin and reflected downwards exposing the extracapsular part of popliteus. Biceps femoris, plantaris and lateral collateral ligament of knee joint were identified at posterolateral corner of knee and divided to expose the tendon of popliteus. The proximal attachments of popliteus to femur, capsule of knee joint, fibular head, lateral meniscus and oblique popliteal ligament were dissected and defined. The distal attachments of popliteus to tibia and fascia over popliteus were also dissected and defined. The arrangement of the muscle fibers of popliteus was also noted. The tibial nerve (TN) was identified at popliteal fossa and nerve to popliteus (NP) was traced till its point of entry into muscle by reflecting it. The number of branches of NP and other muscles supplied by it were noted. A horizontal line connecting medial and lateral femoral epicondyles was considered as intercondylar line (ICL). The point of intersection of the TN and NP at the lower border of popliteus were taken as $\mathrm{A}$ and $\mathrm{B}$ respectively. The following measurements were made (figure $\mathbf{1}$ and figure 2 ):

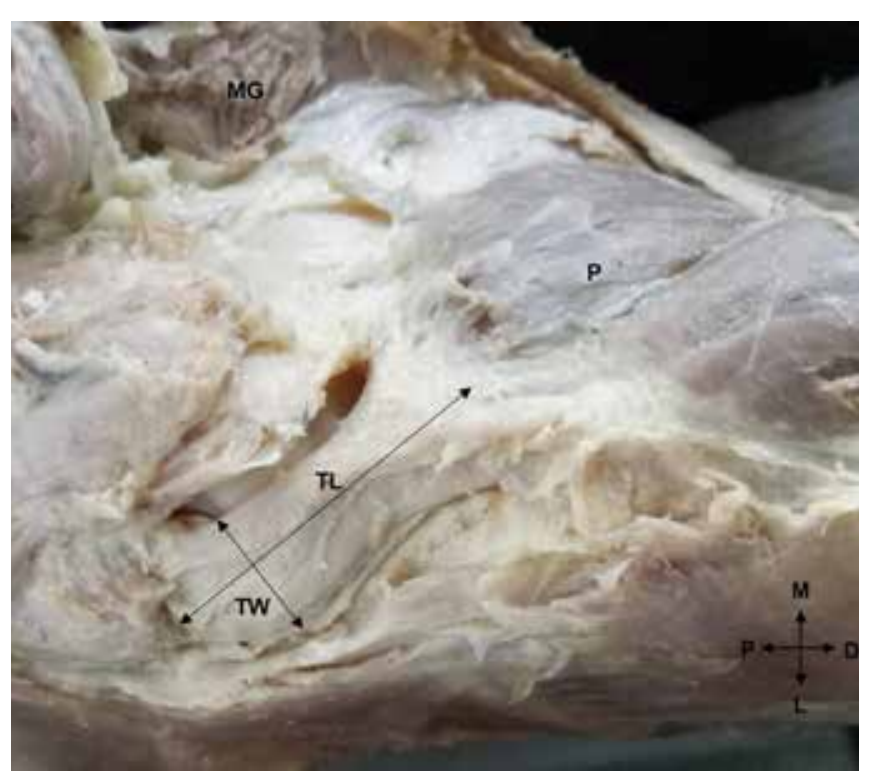

Figure 1 - A photograph of right popliteal region showing the distances measured. $T L$, tendon length; TW, tendon width. (P, popliteus; MG, medial head of gastrocnemius).

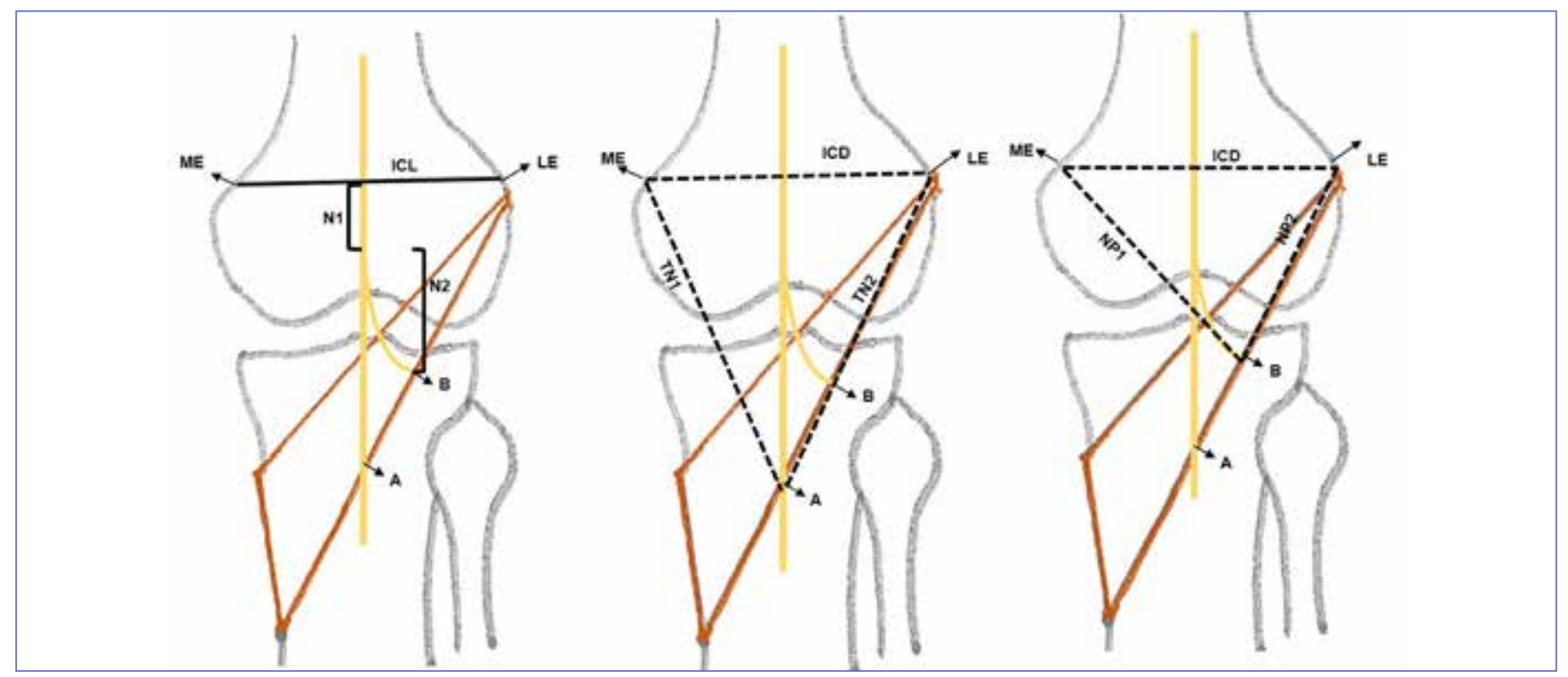

Figure 2 - Schematic diagram of posterior view of right popliteal region showing the distances measured (ME, medial epicondyle; LE, lateral epicondyle; ICL, intercondylar line; A, point of intersection of TN at lower border of popliteus; B, point of intersection of NP at lower border of popliteus). 
1. Tendon length (TL), the distance from the origin of popliteus at lateral femoral condyle to midpoint of its musculotendinous junction;

2. Tendon width (TW), the distance at the widest part of tendon between its medial and lateral border;

3. Muscle length (ML), the distance from the musculotendinous junction of popliteus to its distal attachment on tibia;

4. Muscle width (MW), the distance at the widest part of muscular fibers of popliteus beyond its musculotendinous junction;

5. N1, the distance from the origin of NP to the ICL;

6. N2, the distance from the origin of NP to B;

7. ICD, the distance between the medial and lateral epicondyle;

8. TN1, the distance from the medial epicondyle to A;

9. TN2, the distance from the lateral epicondyle to A;

10. NP1, the distance from the medial epicondyle to $B$;

11. NP2, the distance from the lateral epicondyle to B.

All measurements were made using the digital vernier caliper with accuracy of $0.02 \mathrm{~mm}$ (Aerospace). The measurements were done by the same person twice, to avoid the inter-observer error. Statistical analysis was done using Microsoft Excel. Unpaired student $t$ test was applied to find statistical difference between the measurement of right and left limbs.

\section{RESULTS}

The popliteus tendon had proximal attachment to anterior aspect of groove for popliteus on lateral surface of lateral femoral condyle in all $20(100 \%)$ specimens. The proximal attachments of popliteus to capsule of knee joint, fibular head, lateral meniscus, and oblique popliteal ligament were present in 20 (100\%), 19 (95\%), $11(55 \%)$ and $10(50 \%)$ specimens respectively. The distal attachments of the popliteus to tibia and fascia over popliteus were present in all $20(100 \%)$ specimens. The proximal and distal attachments of popliteus are shown in figure 3. The fibers of popliteus were arranged in a triangular pattern containing oblique fasciculi radiating inferolaterally from the musculotendinous junction of the popliteus.
Table I summarizes the morphometric measurements of the myotendinous complex of popliteus. The ML and MW were between $85.56 \mathrm{~mm}-131.29 \mathrm{~mm}$, and $22.98 \mathrm{~mm}-40.25$ $\mathrm{mm}$, respectively. The TL and TW were between $27.74 \mathrm{~mm}$ - $45.52 \mathrm{~mm}$, and $5.19 \mathrm{~mm}-13.31 \mathrm{~mm}$, respectively. No significant difference was observed in the above parameters between right and left sided specimens.

The NP arose from the TN in all $20(100 \%)$ specimens. The NP from its origin passed deep to plantaris and descended on the posterior surface of popliteus. It supplied the popliteus from its anterior surface after winding around its lower border. The NP also supplied tibialis posterior in $10(50 \%)$ of specimens (figure 4$)$. The NP had one, two, three branches in $10(50 \%), 8(40 \%)$ and $2(10 \%)$ of the specimens respectively. The NP originated from $\mathrm{TN}$ above and below the ICL in $9(45 \%)$ and $11(55 \%)$ specimens.

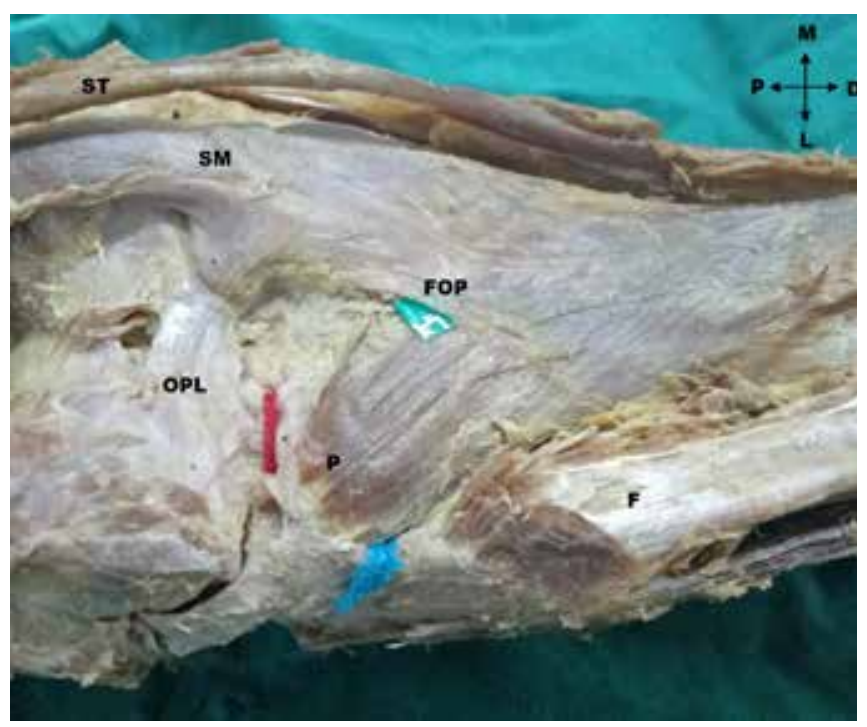

Figure 3 - A photograph of right popliteal region showing the proximal and distal attachments of popliteus. Fibular attachment (Blue), oblique popliteal ligament (red) and fascia over popliteus (Green). (F, fibula; P, popliteus; OPL, oblique popliteal ligament; SM, semimembranosus; ST, semitendinosus).

Table I - The morphometry of the muscle-tendon complex of popliteus.

\begin{tabular}{|c|c|c|c|c|}
\hline \multirow{2}{*}{ Measurement } & \multicolumn{3}{|c|}{ mean $\pm S . D(m m)$} & \multirow{2}{*}{ p-value } \\
\hline & total $(n=20)$ & $\mathrm{R}(\mathrm{n}=10)$ & $L(n=10)$ & \\
\hline TL & $35.12 \pm 5.16$ & $35.46 \pm 5.45$ & $34.77 \pm 5.13$ & 0.774 \\
\hline TW & $9.52 \pm 2.78$ & $10.19 \pm 3.11$ & $8.85 \pm 2.37$ & 0.291 \\
\hline ML & $107.14 \pm 13.45$ & $110.48 \pm 14.43$ & $103.81 \pm 12.20$ & 0.279 \\
\hline
\end{tabular}




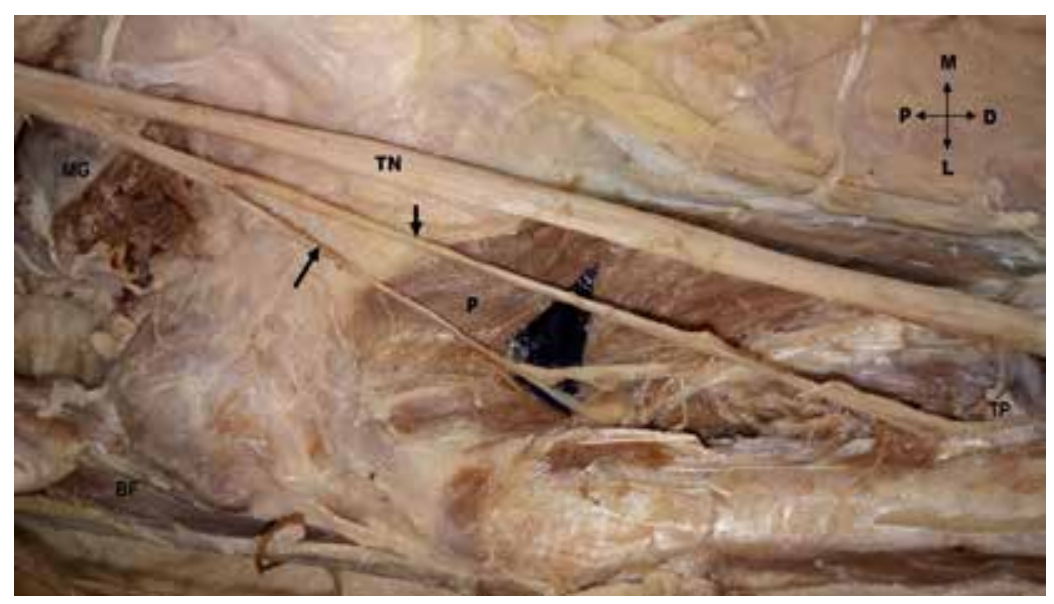

Figure 4 - A photograph of right popliteal region showing the nerve to popliteus (NP) arising from tibial nerve (TN) and nerve to tibialis posterior (NTP) arising from the nerve to popliteus (NP). (P, popliteus; MG, medial head of gastrocnemius; BF, biceps femoris; TP, tibialis posterior).
Table II summarizes the distances measured with respect to origin of the NP. The point of origin of the NP varied between $2.61 \mathrm{~mm}-39.09 \mathrm{~mm}$ above, and $2.16 \mathrm{~mm}-37.47$ $\mathrm{mm}$ below the ICL. In majority $8(88.89 \%)$ and $6(54.54 \%)$ of legs, the point of origin of NP was within $20 \mathrm{~mm}$, above and below the ICL.

Table III shows the distances from the medial and lateral epicondyles to TN and NP at their point of intersection with lower border of popliteus. The TN at lower border of popliteus was located $68.73 \mathrm{~mm}-99.27 \mathrm{~mm}$ from the medial epicondyle, and $69.68 \mathrm{~mm}-98.66 \mathrm{~mm}$ from the lateral epicondyle. In $7(35 \%)$ and $13(65 \%)$ of specimens it was located at $60-80 \mathrm{~mm}$ and $80-100 \mathrm{~mm}$, respectively, from the medial epicondyle. In $9(45 \%)$ and $11(55 \%)$ of specimens it was located at $60-80 \mathrm{~mm}$ and $80-100 \mathrm{~mm}$, respectively, from the lateral epicondyle. The NP at the lower border of popliteus was located at $57.77 \mathrm{~mm}-95.97 \mathrm{~mm}$ from the medial epicondyle, and $40.47 \mathrm{~mm}-98.03 \mathrm{~mm}$ from the lateral epicondyle. In $8(40 \%)$ and $12(60 \%)$ of specimens the NP was located at $50-70 \mathrm{~mm}$ and $70-90 \mathrm{~mm}$, respectively, from the medial epicondyle. In $14(70 \%)$ and $5(25 \%)$ of specimens NP was located at a $50-70 \mathrm{~mm}$ and $70-90$ $\mathrm{mm}$, respectively, from the lateral epicondyle. No significant difference was observed in the above measurements between right and left sided specimens. The ICD measured between $65.41 \mathrm{~mm}-81.53 \mathrm{~mm}$, and there was a statistically significant difference in the observed values of ICD $(\mathrm{p}=$ 0.023 ) between the right and left legs.

Table II - The distances measured with respect to origin of the nerve to popliteus.

\begin{tabular}{ccccccc}
\hline Level of nerve & \multicolumn{3}{c}{$\mathbf{N 1}$} & N2 \\
\hline & $\min (\mathrm{mm})$ & $\max (\mathrm{mm})$ & $\operatorname{mean} \pm \mathrm{SD}(\mathrm{mm})$ & $\min (\mathrm{mm})$ & $\max (\mathrm{mm})$ & $\operatorname{mean} \pm \operatorname{SD}(\mathrm{mm})$ \\
\hline above ICL $(\mathbf{n}=\mathbf{9})$ & 2.61 & 39.09 & $12.10 \pm 10.54$ & 49.18 & 64.66 & $55.68 \pm 4.89$ \\
\hline below ICL $(\mathbf{n}=\mathbf{1 1})$ & 2.16 & 37.47 & $18.74 \pm 11.51$ & 38.64 & 69.62 & $51.35 \pm 8.32$ \\
\hline
\end{tabular}

Table III - The distances measured from the medial and lateral epicondyles.

\begin{tabular}{ccccc}
\hline Measurement & total $(\mathbf{n}=\mathbf{2 0})$ & mean $\pm \mathbf{S D}(\mathbf{m m})$ & $\mathbf{R}(\mathbf{n}=\mathbf{1 0})$ & p-value \\
\hline ICD & $74.99 \pm 4.71$ & $77.37 \pm 2.51$ & $72.60 \pm 5.27$ & 0.023 \\
\hline TN1 & $83.89 \pm 9.12$ & $85.57 \pm 8.68$ & $82.20 \pm 9.69$ & 0.425 \\
\hline TN2 & $82.86 \pm 8.17$ & $82.39 \pm 7.30$ & $83.32 \pm 9.34$ & 0.807 \\
\hline NP1 & $74.07 \pm 9.09$ & $71.39 \pm 8.24$ & $76.76 \pm 9.51$ & 0.194 \\
\hline NP2 & $63.91 \pm 12.27$ & $61.01 \pm 6.19$ & $66.81 \pm 16.15$ & 0.311 \\
\hline
\end{tabular}




\section{DISCUSSION}

Popliteus is one of the smaller muscles with greater functional significance that presents with inversion in its origin and insertion. In present study the popliteus tendon attachment on lateral surface of lateral femoral condyle was present in all $100 \%$ specimens. This observation is in agreement with the classical textbook of Anatomy (5). Total knee arthroplasty is one of the most common surgeries done to relive pain and restore function following damage by arthritis or trauma of the knee joint. Previous studies have showed that the femoral insertion of popliteus tendon could be unavoidably excised during primary total knee arthroplasty (10). Injury to its femoral insertion leads to flexion and extension gaps as well as excessive rotational stress between femur and tibia (10).

The previous studies have also reported the attachment of the popliteus to capsule of knee joint, fibular head, lateral meniscus and oblique popliteal ligament (11-13). The observed frequency of the proximal attachments of popliteus in the present study are compared with the previous studies and are tabulated in Table IV. In present study the attachment to the capsule of knee joint was present in all $100 \%$ specimens. The least of all the observed attachments was to that of oblique popliteal ligament, seen in only $50 \%$ specimens. The frequency of attachment to the meniscus in present study was also less when compared to the previous studies (11-13). The attachments to the arcuate popliteal ligament, ligament of Wrisberg and posterior cruciate ligament were not observed in the present study as reported previously $(12,13)$.

According to Last, the attachments to the capsule and lateral meniscus help to retract the posterior horn during flexion of knee joint, thereby preventing it from injury (1). The attachment to fibula forms the popliteofibular ligament and acts as a single most important stabilizer of the posterolateral region of the knee. It resists lateral rotation of the tibia on the femur (5). The attachment to oblique popliteal ligament might help in strengthening of the postero-lateral knee joint capsule (12).
The shape of popliteus observed was triangular containing oblique fasciculi. Hwang et al has reported the shape of popliteus to be obtuse triangular (6). The fibers are attached at an oblique angle to the resultant line of pull which enables uniform distribution of force over a greater area. The fiber distribution study on human popliteus supports its both tonic regulatory postural control and phasic sudden-position-change functions (3). The previous studies have shown high muscle-spindle concentrations of popliteus with mean spindle density of 7.85 making it to function as a kinesiological monitor (14).

Injury to popliteus is commonly observed as a part of posterolateral corner trauma (2). The popliteal tear can be either intra or extra-articular. Strains commonly affect the muscle belly and myotendinous junction than the tendon (2). The mean length and width of the popliteus tendon in present study was $35.12 \mathrm{~mm}$ and $9.52 \mathrm{~mm}$, respectively. This is almost similar to most of the previous studies $(15,16)$. The length of popliteal tendon in present study was found to be shorter than that reported by Kurtoglu et al (17). The mean length and width of the muscle belly was $107.14 \mathrm{~mm}$ and $32.38 \mathrm{~mm}$, respectively. The study by Hwang et al showed the length along the lateral border of the triangular popliteus was $11.9 \pm 1.5 \mathrm{~cm}$, which is almost similar to length observed in present study (6). PMTC is also used as a landmark in sling reconstruction of popliteus tendon (4).Thus, the knowledge about the PMTC morphometry can aid during popliteus reconstruction surgeries.

The classical textbooks in Anatomy describe the TN branch to popliteus as descending obliquely across the popliteal vessel and curling around the distal border of the muscle on its anterior surface, and then supplying it from anterior surface. The origin and course of NP in present study was similar to the above literature. But it was different from that reported in a previous study, which stated that the nerve branches ran superficially on the periosteum of the tibia and entered the popliteus on its superficial surface about $1 \mathrm{~cm}$ distal to its superior border (6). According to literatures, the NP also gives a twig to tibialis posterior (18).

Table IV - Comparison between the various studies on the proximal attachment sites of popliteus.

\begin{tabular}{|c|c|c|c|c|}
\hline Attachment sites & $\begin{array}{l}\text { Simonnet et al., } 2003 \\
\text { frequency } \%(n=42)\end{array}$ & $\begin{array}{l}\text { Paraskevas et al., } 2006 \\
\text { frequency } \%(n=40)\end{array}$ & $\begin{array}{c}\text { Upasna, } 2014 \\
\text { frequency } \%(n=60)\end{array}$ & $\begin{array}{c}\text { present study } \\
\text { frequency } \%(n=20)\end{array}$ \\
\hline capsule & 57 & 100 & - & 100 \\
\hline menisci & 95 & 91.67 & 100 & 55 \\
\hline oblique popliteal ligament & 79 & - & 40 & 50 \\
\hline
\end{tabular}


But in the present study the twig to tibialis posterior arising from NP was present in only $50 \%$ of specimens differing from the above. The point of origin of NP above and below the ICL was within $40 \mathrm{~mm}$ in the present study. This knowledge about NP is important during nerve blocking process to relieve in-toeing gait in patients with spasticity of the lower limb (6).

The minimum distance of the TN at lower border of the popliteus from medial and lateral epicondyle was $68.73 \mathrm{~mm}$ and $69.68 \mathrm{~mm}$, respectively. The minimum distance of the $\mathrm{NP}$ at lower border of the popliteus from medial and lateral epicondyle was $57.77 \mathrm{~mm}$ and $40.47 \mathrm{~mm}$, respectively. Therefore, $65 \mathrm{~mm}$ and $40 \mathrm{~mm}$ from the medial and lateral epicondyle can be considered as safe zone for the TN and NP at lower border of popliteus (figure 5). This data has not been reported in the previous literatures to the best of our knowledge. The safe zone for TN and NP would be useful in surgeries using a posterior knee joint approach for treating tibial plateau fractures, baker's cysts and posterior horn or root meniscal tears.

The limitations of present study include relatively small sample size and shrinkage related to embalming procedure.
The gender differences also could not be assessed. The present study didn't encounter any variations in PMTC, and hence the morphometry related to variations weren't included.

We believe the present study has provided additional data on the morphometry and nerve supply of myotendinous complex of popliteus. The morphology and morphometry of PMTC can aid during arthroplasty and popliteal reconstruction surgeries. The safe zone of TN and NP would help during knee joint surgeries with posterior approach. Thus, the present study may act as an anatomical guide to the orthopedic surgeons.

\section{Acknowledgements}

The authors would like to acknowledge those who had donated the bodies for scientific purpose through the body donation program.

\section{Conflicts of interest}

The authors declare no conflict of interest for the present study.

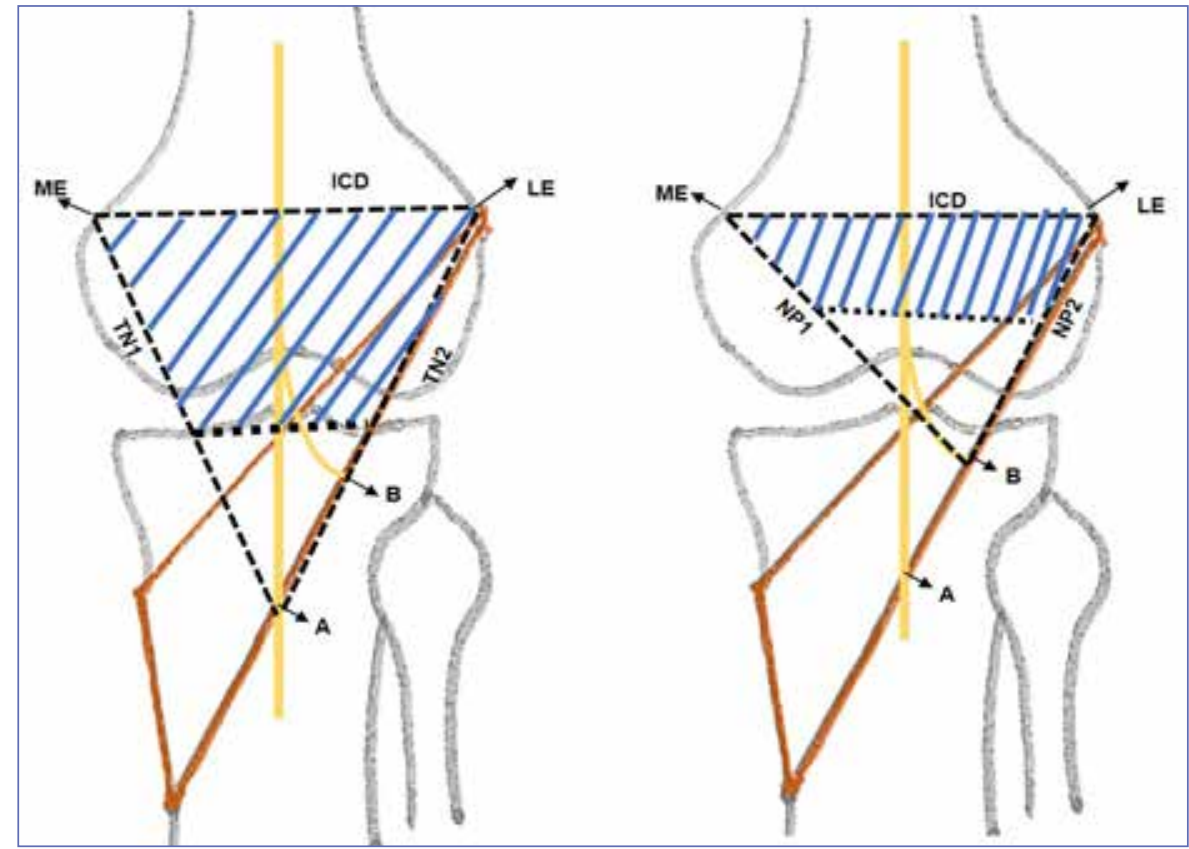

Figure 5 - Schematic diagram of posterior view of right popliteal region showing the safe zone (blue color) for the tibial nerve (TN) and nerve to popliteus (NP). (ME, medial epicondyle; LE, lateral epicondyle; $I C L$, intercondylar line; $A$, point of intersection of $\mathrm{TN}$ at lower border of popliteus; B, point of intersection of NP at lower border of popliteus). 


\section{REFERENCES}

1. Last RJ. The popliteus muscle and the lateral meniscus. J Bone Joint Surg Am 1950; 32:93-9.

2. Jadhav SP, More SR, Riascos RF, Lemos DF, Swischuk LE. Comprehensive review of the anatomy, function, and imaging of the popliteus and associated pathologic conditions. RadioGraphics 2014; 34(2):496-513.

3. Nyland J, Lachman N, Kocabey Y, Brosky J, Altun R, Caborn D. Anatomy, function, and rehabilitation of the popliteus musculotendinous complex. J Orthop Sports Phys Ther 2005; 35(3):165-179.

4. Feng H, Hong L, Geng X, Zhang H, Wang X, Zhang J. Posterolateral sling reconstruction of the popliteus tendon: an all-arthroscopic technique. Arthroscopy: The Journal of Arthroscopic and Related Surgery 2009; 25(7):800-805.

5. Stranding S, Borley NR, Gray H. Gray's anatomy: the anatomical basis of clinical practice. 41st ed. Edinburgh: Churchill Livingstone/Elsevier; 2016:1397.

6. Hwang K, Lee KM, Han SH, Kim SG. Shape and innervation of popliteus muscle. Anatomy and Cell Biology 2010; 43(2):165-168.

7. Faucett SC, Gannon J, Chahla J, Ferrari MB, LaPrade RF. Posterior surgical approach to the knee. Arthroscopy Techniques 2017; 6(2):e391-395.

8. Cunningham DJ. The lower limb. In: Romanes GJ (ed). Cunningham's manual of practical anatomy, upper and lower limbs. 15th ed. Oxford: Oxford university press; 1986:160-190.

9. Sergio C, Claudia G, Paola C, Marta AS et al. Clinical evidence of traditional vs fast track recovery methodologies after total arthroplasty for osteoarthritic knee treatment. A retrospective observational study. Muscles, Ligaments and Tendons Journal 2017; 7(3):504-513.
10. Takahashi A, takahashi S, toshimi A, Dalsuke C et al. Potential risk of excising the femoral insertion of the popliteus tendon during primary total knee arthroplasty: a biometric study. J Orthop Sci 2015; 20:1030-1035.

11. Paraskevas G, Papaziogas B, Kitsoulis P, Spanidou S. A study on the morphology of the popliteus muscle and arcuate popliteal ligament. Folia morphologica 2006; 65(4):381-384.

12. Simonnet M-L, Rooze M, Feipel V. The proximal attachments of the popliteus muscle: a quantitative study and clinical significance. Surgical and Radiologic Anatomy 2003; 25(1):58-63.

13. Upasna AK. Proximal attachments of popliteus Muscle: A morphological study on 30 adult human cadavers. JIMSA 2014; 27(1):19-20.

14. Peck D, Buxton DF, Nitz A. A comparison of spindle concentrations in large and small muscles acting in parallel combinations. J Morphol 1984; 180(3):243-252.

15. Jung GH, Kim JD, Kim H. Location and classification of popliteus tendon's origin: cadaveric study. Arch Orthop Trauma Surg. 2010; 130(8):1027-1032.

16. Osti M, tschann P, Künzel KH, Benedetto KP. Posterolateral corner of the knee: microsurgical analysis of anatomy and morphometry. Orthopedics 2013; 36(9):e1114-1120.

17. Kurtoglu Z, Elvan O, Aktekin M, Çolak M. Morphological features of the popliteus tendon, popliteofibular and lateral (fibular) collateral ligaments. International Journal of Morphology 2017; 35(1):62-71.

18. Datta AK. Esssentials of Human Anatomy (vol 3) Superior and Inferior Extremities. 4th ed. Current Books Int; Kolkota 2010:197. 